\title{
Isolation and analysis of a very virulent Marek's disease virus strain in China
}

\author{
Zhenhua Gong ${ }^{1 *}$, Lijuan Zhang ${ }^{1}$, Jianlin Wang ${ }^{2}$, Linlin Chen², Hu Shan², Zhiliang Wang ${ }^{1}$ and Hongchao Ma
}

\begin{abstract}
Background: A severe MD was broken out at a farm in Shandong, China, despite FC126 vaccination of the chickens at 1-day-old. The mortality of the flocks reached up to $38.3 \%$. The infected chickens were found to have MD pathological changes, including enlargement of spleens, livers and kidneys, and tumors occured on organs later. Samples were collected from the chickens for diagnosis.

Methods: The collected samples were inoculated into primary duck embryo fibroblast (DEF) cells, and the MDV strain named SD2012-1 was isolated. In order to identify the isolate, amplification by PCR and sequencing of oncogenic Meq and VIL-8 gene were processed, the obtained sequences were compared with the sequences of reference strains, and SD2012-1 was used to challenge immunized SPF chickens.

Results: A very virulent MDV isolate strain, SD2012-1, was isolated from a chicken flock in Shandong Province, China, the isolate had the characteristics of very virulent MDV-1, nucleotide and deduced amino acid sequence comparisons of Meq and VIL-8 gene of SD2012-1 with those of reference strains showed SD2012-1 had high homology with MDV strains isolated from China, SD2012-1 could break through the protection provided by HVT vaccine and $\mathrm{HVT}+\mathrm{SB}-1$ vaccine immunization and caused the mortality of SPF chickens over $60 \%$. The immune failure occured at the farm could be due to the improper selection of vaccines. SD2012-1 produced death later and the gross postmortem lesions of chickens died early and later were different.

Conclusions: MDV strain SD2012-1 isolated from Shandong Province, China was found to have the characteristics of very virulent MDV-1, which could break through the protection provided by HVT vaccine and HVT + SB-1 vaccine, the virus seemed to have a long latent period, and cause different gross postmortem lesions of chickens between chickens died early and later. A better immunization way should be chosen to prevent infection of this MDV strain in field.
\end{abstract}

Keywords: Marek's disease virus, Isolation, PCR, Oncogenic gene, Virulence, Immunization

Marek's Disease Virus (MDV) was an oncogenic poultry herpesvirus, causing not only death of chickens directly, but also immunosuppression of infected chickens sensitive to other pathogens, MDV strains (MDVs) had become increasingly virulent since the 1960s [1,2]. MDVs were classified into three serotypes which had major differences in genome and biological features. Serotype 1 MDVs included all the oncogenic strains and their attenuated forms; serotype 2 MDVs were non-oncogenic viruses isolated in chickens; serotype 3 MDVs were nononcogenic viruses isolated in turkey, generally known as

\footnotetext{
* Correspondence: gzh1832@126.com

'China Animal Healthy and Epidemic Center, Qingdao 266032, China

Full list of author information is available at the end of the article
}

herpesvirus of turkey or HVT [3]. serotype 1 MDVs could be further classified into four pathotypes, including mild $(\mathrm{m})$, virulent $(\mathrm{v})$, very virulent (vv) and very virulent plus $(\mathrm{vv}+)$ strains $[4,5]$.

Control of Marek's disease was predominantly via vaccination of chickens, three types of vaccine had been developed for use against MD. These were herpesvirus of turkey (HVT), non-pathogenic serotype $2 \mathrm{MDV}$ and non-pathogenic serotype 1 . In 1970s, HVT vaccine was mainly used to control the disease. In mid-1980s, serotype 2 vaccine such as strain SB-1 was used in combination with HVT against the enhanced virulence strains. With further increase in virulence of field viruses [6], serotype 1 vaccine CVI988/Rispens [6,7] was introduced
Ciomed Central

(c) 2013 Gong et al.; licensee BioMed Central Ltd. This is an Open Access article distributed under the terms of the Creative Commons Attribution License (http://creativecommons.org/licenses/by/2.0), which permits unrestricted use, distribution, and reproduction in any medium, provided the original work is properly cited. 
for widespread use in the 1990s. Recently, the failure of CVI988/Rispens vaccination had been reported, when used either alone or in combination with serotype 2 and/or serotype 3 vaccines, suggesting the emergence of high virulent strains $[8,9]$.

In recent years, there were virulent MDVs circulating in China, the mortality rate of MD could reach up to $15 \%-60 \%$. Isolate of MDVs in China were reported in breeder or layer flocks which had been vaccinated by HVT or CVI988/Rispens [10-12], the isolates were high pathogenic for chickens, the emergence of MDVs of increasing virulence was a significant problem for the poultry industry.

\section{Methods}

\section{Samples collection}

A severe MD was broken out at a farm with 3000 chickens in Shandong, China, despite FC126 vaccination of the chickens at 1-day-old. The clinic symptoms and death of MD in the chicken flocks began at about 60day-old and continued until 135-day-old, the mortality of the flocks reached up to $38.3 \%$. The infected chickens were found to have MD pathological changes, including enlargement of spleens, livers and kidneys, and tumors occured on organs later. Heparinised blood samples were collected from chickens with tumor lesions for diagnosis.

This experimental research on animals were performed with the approval of Experimental Animal Administrative Center of Shandong Province.

\section{Virus isolation}

The collected samples were kept under refrigeration and transported, lymphocytes separated from the blood samples by lymphocyte separation medium were inoculated into primary duck embryo fibroblast (DEF) cells prepared from 11-day-old embryonated eggs, the inoculated DEF cells were incubated at $37^{\circ} \mathrm{C}$ with $5 \% \mathrm{CO} 2$ for five days. After 3 passages, typical cytopathic effect (CPE) of the inoculated DEF cells stabilized at 96 hrs after inoculation. The MDV strain named SD2012-1 was obtained by isolation, and the DEF cell cultures of SD2012-1 were collected and stored at $-196^{\circ} \mathrm{C}$ in $10 \%$ dimethyl sulphoxide.

\section{PCR amplification of oncogenic genes}

The DNA of SD2012-1 as PCR templates was extracted from inoculated DEF culture samples and tissue samples with tumor lesions using mini-spin column chromatography method. In brief, samples was treated using proteolytic enzyme, then an equal volume of $100 \%$ ethanol was added to the lysate and the DNA was purified by mni-spin column chromatography. Contaminating proteins were removed via ethanol/salt washes, and the DNA was eluted for PCR.

The MDV target genes of PCR were Meq and vIL-8 gene, which were reported to have the greatest possibility being associated with viral oncogenicity and pathogenicity [1]. Primers for Meq and vIL-8 gene had been used to amplify the same genes of $18 \mathrm{MDV}$ isolates from China [11], the PCR product of Meq and vIL-8 gene of SD2012-1 were produced individually by amplification [11] of the virus DNA extracted from DEF cultures and tissue samples infected with SD2012-1.

In order to verify the absence of infection of chickens with avian leukosis virus (ALV) and reticuloendotheliosis virus (REV), all the samples for amplification of MDV Meq and vIL- 8 genes were also detected by previously reported PCR method for ALV [13] and REV [14].

The primers which had been used for PCR amplification of MDV, ALV and REV were shown in Table 1.

\section{DNA cloning and sequencing of SD2012-1 strain}

The PCR products of Meq gene and vIL-8 gene of SD2012-1, corresponding to the predicted size, were purified from agarose gel, and were ligated with a TA cloning vector $\mathrm{pMD19-T}$, and then were transformed into DH5a competent cell individually. The cloned Meq and vIL-8 genes were then sequenced by Sanger dideoxy sequencing method.

\section{Comparison of Meq and vIL-8 gene of SD2012 with reference strains}

The obtained Meq and vIL-8 nucleotide sequence and the deduced amino acid sequence of SD2012-1 were compared with 20 reference MDVs for the homology analysis with the use of MegAlign program. Among these 20 reference strains, 3 strains were vaccine strains, 11 strains were isolated from China, and 6 strains were

Table 1 Primers for MDV Meq and vIL-8 genes, for ALV and REV

\begin{tabular}{|c|c|c|c|}
\hline Virus/Gene & Primer se & & DNA sizes \\
\hline Meq & F: 5'-GGCACGGTACAGGTGTAAAGAG-3' & R: 5'-GCATAGACGATGTGCTGCTGAG-3' & $1081 \mathrm{bp}$ \\
\hline VIL-8 & F: 5'-GAGACCCAATAACAGGGAAATC-3' & R: 5' -TAGACCGTATCCGTGCTCCATC-3' & $886 \mathrm{bp}$ \\
\hline ALV & F: 5'-AATTCTGCTTGAAATATG-3' & R: 5'-AGTTGTCAGGGAATCGA-3' & $436 \mathrm{bp}$ \\
\hline REV & F: 5'-CATACTGGAGCCAATGGTGTAAAGGGCAGA-3' & R: 5'-AATGTTGTAGCGAAGTACT-3' & $291 \mathrm{bp}$ \\
\hline
\end{tabular}


isolated from USA. 20 strains were used for comparison of Meq genes, and 8 strains were used for comparison of vIL-8 genes. The MDV reference strains were retrieved from the GenBank database, and the backgrounds of the reference strains used in this study were listed in Table 2.

\section{Virulence studies of SD2012-1}

90 SPF chickens randomly divided into three groups were vaccinated at the age of one day, In group 1, 30 chickens were vaccinated with 2000 PFU HVT (FC126 strain, 2000 PFU HVT had been the suggested dosage used at the farm with MD broken out); in group 2, 30 chickens were vaccinated with bivalent vaccine (2000 PFU FC126 + 1200 PFU SB-1); in group 3, 30 chickens were inoculated with phosphate buffer. At the age of ten days, all the chickens were challenged intra-abdominally with $0.5 \mathrm{ml}$ diluent of plaque purified SD2012-1 strain containing 4000 PFU. Clinical signs and gross postmortem lesions were recorded after virus challenge. Organs of chickens with lesions were sampled for PCR diagnosis.

\section{Results}

PCR amplification for Meq and vIL-8 gene of SD2012-1

By PCR [11] of the extracted virus DNA with primers in Table 1, the specific presence of an approximately $1081 \mathrm{bp}$ long DNA product for MDV Meq gene (Figure 1-A) and the specific presence of an approximately 886 bp long DNA product for MDV vIL-8 gene (Figure 1-B) were found in samples, including the DEF cultures infected with SD2012-1 isolate, samples collected from chickens infected with SD2012-1 at the farm and samples collected from challenged chickens with SD2012-1, the PCR products from all the samples were obvious, which verified the existence of infection with MDV to chickens and inoculated DEF cells.

\section{Detection of ALV and REV by PCR}

All the samples, including the DEF cultures infected with SD2012-1 isolate, samples collected from chickens infected with SD2012-1 at the farm and samples collected from challenged chickens with SD2012-1were verified to be negative of Avian Leukosis virus (Figure 1-C) and Reticuloendotheliosis virus (Figure 1-D) by PCR, which showed the absence of ALV and REV in these samples.

\section{sequence of Meq and vlL-8 gene of SD2012-1}

By sequencing, the nucleotide sequence of Meq and vIL-8 gene of SD2012-1 and their deduced amino acid sequence were obtained, the sequences of SD2012-1 were submitted to the GenBank database with the accession

Table 2 MDV reference strains published in GenBank

\begin{tabular}{|c|c|c|c|c|}
\hline MDV strains & Virulence & Geographic origin & Year of isolation & Accession number \\
\hline 0093 & High virulence & Guangxi, China & 2002 & AF493550(M) \\
\hline 0095 & High virulence & China & 2002 & AF493552(M) \\
\hline 0297 & High virulence & China & 2002 & AF493553(M) \\
\hline 0304 & High virulence & Guangxi, China & 2002 & AF493554(M) \\
\hline $\mathrm{G} 2$ & High virulence & Guangxi, China & 2002 & AF493556(M) \\
\hline GX070060 & High virulence & China & 2008 & EU427303(M) \\
\hline GX070079 & High virulence & China & 2008 & EU427304(M) \\
\hline GXY2 & High virulence & China & 2007 & EF546430(M) \\
\hline YLO40920 & High virulence & China & 2005 & DQ174459(M) \\
\hline LS & High virulence & Sichuan, China & 2008 & HQ638149(M, V) \\
\hline LMS & High virulence & Sichuan, China & 2007 & HQ658622(M, V) \\
\hline 3004 & Vaccine strain & Russia & $\mathrm{N} / \mathrm{A}$ & EU032468(M) \\
\hline 814 & Vaccine strain & China & N/A & GU354326(M) \\
\hline CVI988 & Vaccine strain & Netherland & 1972 & DQ534538(M, V) \\
\hline $\mathrm{CU}-2$ & Mild virulence & USA & N/A & EU499381(M, V) \\
\hline GA & virulence & USA & 1964 & AF147806(M), AF065430(V) \\
\hline Md5 & Very virulenc e & USA & 1979 & AF243438(M, V) \\
\hline RB1B & Very virulence & USA & 1982 & EF523390(M, V) \\
\hline $648 \mathrm{~A}$ & Very virulence plus & USA & 1997 & AY362725(M), DQ534534(V) \\
\hline $584 \mathrm{~A}$ & Very virulence plus & USA & Before 2000 & DQ534532(M, V) \\
\hline
\end{tabular}

" $\mathrm{M}$ " refers to accession number of Meq gene; "V" refers to accession number of vlL-8 gene; "N/A" refers to data not available. 

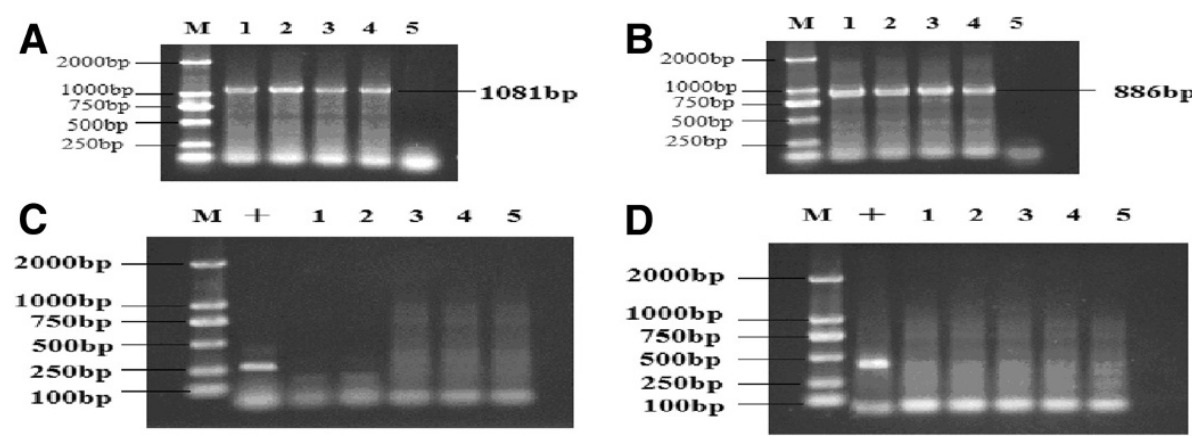

Figure 1 Detection of MDV, ALV and REV by PCR. (A) PCR amplification of Meq gene; (B) PCR amplification of Vil-8 gene. (C) Detection of ALV by PCR; (D) Detection of REV by PCR; (M) DL 2000 DNA Marker; (+) Positive control of ALV in (C) or REV in (D). (1) Spleen of infected chicken from farm with MD broken out; (2) DEF culture infected with MDV SD $2012-1$; (3) Spleen of 55-day-old died chicken without tumor; (4) Spleen of 90-day -old chicken with tumor; (5) DEF culture without infection.

number KC511815 for Meq gene and KC511812 for vIL-8 gene.

\section{Sequence analysis of SD2012-1}

Comparisons of Meq and vIL-8 gene of SD2012-1 with those of reference strains showed SD2012-1 had high homology with the reference strains.

Comparisons of the nucleotide sequence of Meq gene of SD2012-1 with the sequence of 20 reference strains in Table 2 showed the nucleotide homology of SD2012-1 isolate with the 20 strains was between 94.7-99.8\%, SD2012-1 had the highest homology with LS at $99.8 \%$, and had the lowest homology with CVI988 at $94.7 \%$.

The Meq amino acid of SD2012-1 was 339aa long, comparisons of Meq protein amino acid sequence of SD20121 with the sequence of 20 strains in Table 2 showed the amino acid homology of SD2012-1 isolate compared with the 20 strains was between 96.5-99.4\%, SD2012-1 had the highest amino acid homology with LS at $99.4 \%$, and had the lowest homology with CVI988 at 96.5\%. As most strains isolated in China, SD2012-1 had the same amino acid mutation of Meq protein (Figure 2) at position 71 ( $\mathrm{S}$ to $\mathrm{A}$ ), 77(K to E), 80(D to $\mathrm{Y}$ ), 115(V to $\mathrm{A}$ ), 119(R to $\mathrm{C}$ ), 153(Q to $\mathrm{P}$ ), 176( $\mathrm{P}$ to $\mathrm{R}$ ) and 217(P to $\mathrm{A}$ ), except no mutation of SD2012-1 at position 139(remain T).

Comparison of the nucleotide sequence of vIL- 8 gene of SD2012-1 with the sequence of 8 strains, CVI988, CU-2, GA, RB1B, 648A, 584A, LS, LMS, showed the nucleotide homology of SD2012-1 isolate with 8 reference strains were between 99.4-99.6\%, SD2012-1 had the highest homology with LS, RB1B and CU-2 at 99.6\%, and had the lowest homology with CVI988 and 584A at 99.4\%.

\begin{tabular}{|c|c|c|c|c|c|c|}
\hline & 70 & 80 & 110 & 150 & 180 & 220 \\
\hline SD2012-1.pro & DAAR & 2TYYVDKLHEICEE & RTECTSLRAQLACHEPV & LLITPHDPVPEPPICTPPPPS: & TPRPPDTEEL & 'PICTPAPPDAEE: \\
\hline $009.3 \mathrm{MEQ} \cdot$ pro & DAAR & QTYYVDKLHEACEE & RTECTSLRAQLACHEPV & LIIIPHDPVPEPPICIPPPPS: & IPRPPDTEEL & 'PICTPAPPDAEE: \\
\hline $0095 \mathrm{MEQ}$.pro & DAAR & QTYYVDKLHEACEE & RTECTSLRAQLACHEPV & LLTTPHDPVPEPPICTPPPRS: & TPRPPDTEEL & 'PICTPAPPDAEE: \\
\hline $0297 \mathrm{MEQ} \cdot$ pro & DARR & QTYYVDKLHEACEE & RTECTSLRAQLACHEPV & LLTAPHDPVPEPPICTPPPPS: & TPRPPDTEEL & 'PICTPAPPDAEE: \\
\hline 0304 MEQ.pro & DAAR & QTYYVDKLHEACEE & RTECTSLRAQLACHEPV & LLIAPHDPVPEPPICTPPPPS: & IPRPPDTEEL & 'PICT PAPPDAEE: \\
\hline 584A MEQ.pro & DAAR & QFPYVDKLHEACEE & RTECTSLBVRLIPAPEV & LLITPHDPVPEPPICT H PPS: & IAPPPDTEEL & 'PICTPAPPDAEE: \\
\hline 648A MEQ.pro & DAAR & RIEYVDLKLHEACEE & RTECTSLBVVRLABHEPV & LLIT PHDPVPEPPICT HCPS: & IAPPREAEL & 'PICTPAPPDAEE' \\
\hline CU-2 MEQ.pro & DASB & QI LYVDKL HEACEE & RTECTSLBVVLLACHEPV & LLITPHDPVPEPPICIPPPPS: & IAPPPDTEEL & ICTPAFEDAE L \\
\hline CVI988 MEQ.pro & DASR & QIDYVDKLHEACEE & RTECTSLEWVLACHEPV & LLTTPHDPVPEPPICTPPPPS: & TAEPRDTEEL & "PICTHEPPDAEE: \\
\hline DY01 Meq gene.pro & DAAR & QTYYVDKLHEACEE & RTECTSLRAQLACHEPV & LLTAPHDPVEPPICTPPPPS: & TPRPPDTEEL & 'PICT PAPPDAEE: \\
\hline DY04 Meq gene.pro & DAR & QTYYVDKLHEACEE & RTECTSLRAQLLACHEPV & LLIAPHDPVPEPPICTPPPPS: & IPRPPDTEEL & 'PICTPAPPDAEE: \\
\hline G2 MEQ.pro & DAAR & QTYYVDKLHEACEE & RTECTSLRARLACHEPV & LLTTPHDPVPEPPICT PPPPS: & IAPPPDTEEL & 'PICTPAPPDAEE: \\
\hline GA MEQ.pro & DAAR & REFIVDKLHEACEE & RTECTSLEVRLARCHEPV & LLITPHDPVPEPPICTPPPPS: & TAEPRDTEEL & 'PICT HFPPDAEE: \\
\hline GX070060 MEQ.pro & DAAR & QTYYVDKLHEACEE & RTECTSLRAQLACHEPV & LLIIAPHDPVPEPPICT PPPPS: & TPRPPDTEEL & 'PICT PAP PDAEE: \\
\hline GX070079 MEQ.pro & DARR & QTYYVDKLHEACEE & RTECTSLRAQLACHE PW & LLIAPHDVVEPPICT PPPPS: & TPRPPDTEEL & 'PICT PAP PDAEE: \\
\hline GXY2 MEQ.pro & DAAR & QTYYVDKLHEACEE & RTECTSLRAQLACHEPV & LLIAPHDPVPEPPICT PPPPS: & TPRPPDTEEL & 'PICTPAP PDAEE: \\
\hline LS Meq gene.pro & DAAR & QTYYVDKLHEACEE & RIECTSLRAQLACHEPV & LLTTPHDPVPEPPICTPPPPS: & IPRPPDTEEL & 'PICT PAPPDAEE: \\
\hline Md5 MEQ.pro & DAAR & QHFYVIKLHEACEE & RTECTSLEVVILACHEPV & LLTTPHDPVPEPPICTPPPPS: & IAPPPDTEEL & 'PICTPAPPDAEE: \\
\hline RB1B MEQ.pro & DAAR & RAEYYVDKLHEACEE & RTECTSLRVVLACHEPV & LLITPHDPVPEPPICTPPPPS: & TAEPPDTEEL & 'PICT HEPPDAEE: \\
\hline LMSMeq gene.pro & DAAR & QTYYVDKLHEACEE & RTECISLRAQLACHEPV & ILIAPHDPVPEPICIPPPPS: & TPRPPDTEEL & 'PICTPAPPDAEE: \\
\hline YL040920 gene.pro & & QTYYVDKLHEACEE & RTECTSLRAQLACHEPV & LLIABDPVPEPPICT PPPPS: & TPRPPDTEEL & 'PICTPAPPDAEE: \\
\hline
\end{tabular}




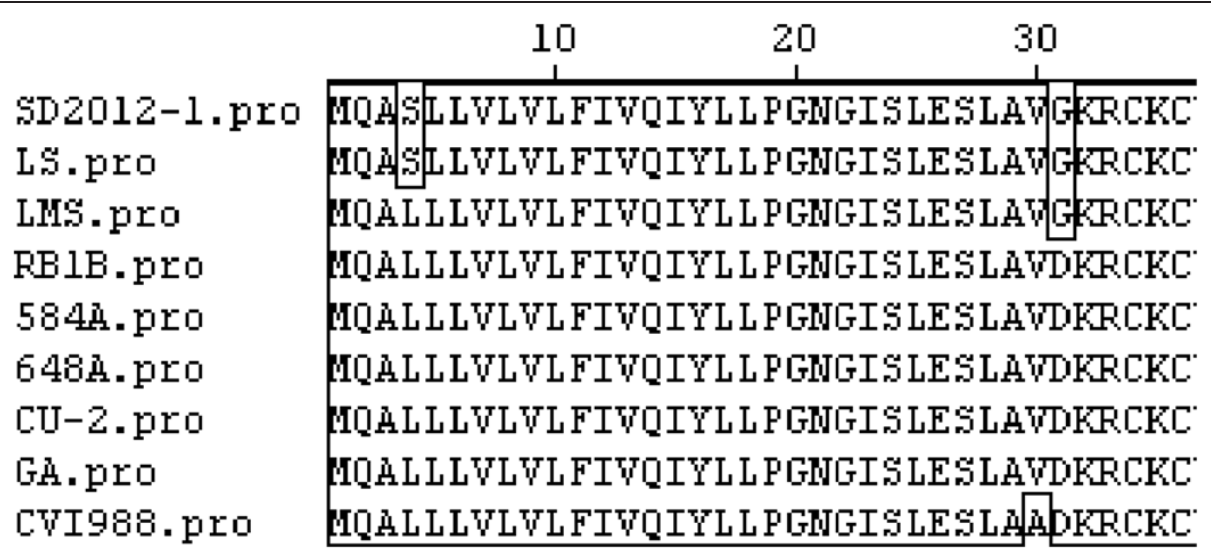

Figure 3 Alignment for amino acid mutation site on vlL-8 gene of 9 MDVs.

The amino acid of SD2012-1 was 134aa long, comparison of vIL-8 protein amino acid sequence of SD2012-1 with CVI988, CU-2, GA, RB1B, 648A, 584A, LS and LMS showed that the amino acid homology of SD2012-1 isolate with 8 strains was between 97.8-99.3\%, SD2012-1 had the highest homology with LS at 99.3\%, and had the lowest homology with CVI988 at $97.8 \%$. The vIL-8 gene amino acid sequence of CU-2, GA, RB1B, 648A, 584A were conservative without amino acid mutation between the 5 strains. As LS did, SD2012-1 had the same amino acid mutation of vIL-8 protein (Figure 3) at position 4(L to S) and 31(D to G) compared with CU-2, GA, RB1B, 648A, $584 \mathrm{~A}$.

\section{Virulence of SD2012-1}

No clinical sign was observed during the first 6 week post challenge of SD2012-1. The earliest death appeared on 45 days post challenge in no vaccine group, and on 48 days post challenge in HVT immunized group and $\mathrm{FC} 126+$ SB-1 group. The peak of death came on 60-85 days post challenge. The mortality were $70.3 \%$ in no vaccine group, $66.7 \%$ in HVT immunized group and 60\% in FC126 + SB1 immunized group (Table 3). Organic samples collected from the chickens with lesions were detected to be MDV positive by PCR (Figure 4). the main gross lesion of the early died chickens were degeneration and swelling of organs without development of tumor (Figure 5). The gross lesion of the later died chickens were mainly tumors

Table 3 Statistics of chickens challenged with MDV $\mathrm{SD}_{\text {2012-1 }}$ isolate

\begin{tabular}{ccccc}
\hline Group & $\begin{array}{c}\text { Bird } \\
\text { number }\end{array}$ & $\begin{array}{c}\text { Death with } \\
\text { tumor }\end{array}$ & $\begin{array}{c}\text { Tumor } \\
\text { lesion }\end{array}$ & Total (\%) \\
\hline no vaccine & 30 & 9 & 13 & $22(70.3 \%)$ \\
HVT & 30 & 8 & 12 & $20(66.7 \%)$ \\
$H V T+$ SB1 & 30 & 8 & 10 & $18(60 \%)$ \\
\hline
\end{tabular}

developed on liver, heart and mesentery, and gizzard swelling with thicken corneum layer which was easy to be peeled (Figure 6).

\section{Discussion}

In the past 40 years, the virulence of MDVs had been increasing gradually, which was possibly caused by the selective pressure of vaccination [5,15-18]. With isolation and gene sequencing, the oncogenic gene changes emerged in the predominant strains were monitored, and the effectiveness of existing vaccines were evaluated [19], both played an important role on MD prevention. In recent years, MD occurred frequently in China in spite of vaccination, a number of MDVs were isolated from different area in China, molecular epidemiologic studies on the amino acid sequences of oncogenic gene of MDVs indicated that the amino acid mutations in Meq gene and vIL8 gene displayed perfect regularity in MDVs circulating in China, which could be considered as features of field MDVs prevalent in recent years in China $[11,12,20]$.

In this study, a virulent MDV isolate which caused severe death of chicken at the infected farm was isolated by transfecting monolayer of duck embryo fibroblast (DEF) with the peripheral blood lymphocytes (PBLs) of the infected chicken, the MDV isolate strain named SD2012-1 was isolated from a chicken flock in Shandong Province, China, which had been vaccinated with HVT vaccine, the isolate was found to have the characteristics of virulent MDV-1.

The oncogenic Meq gene and vIL-8 gene of SD2012-1 were amplified by PCR and sequenced. Compared with 20 reference strains, SD2012-1 had the highest Meq nucleotide sequence homology with LS, a virulent strain isolated from China, at $99.8 \%$, and had the lowest nucleotide sequence homology with CVI988 at $94.7 \%$. As most strains isolated in China [11], the Meq amino acid of SD2012-1 was 339aa long, which was 59aa shorter than Meq protein 


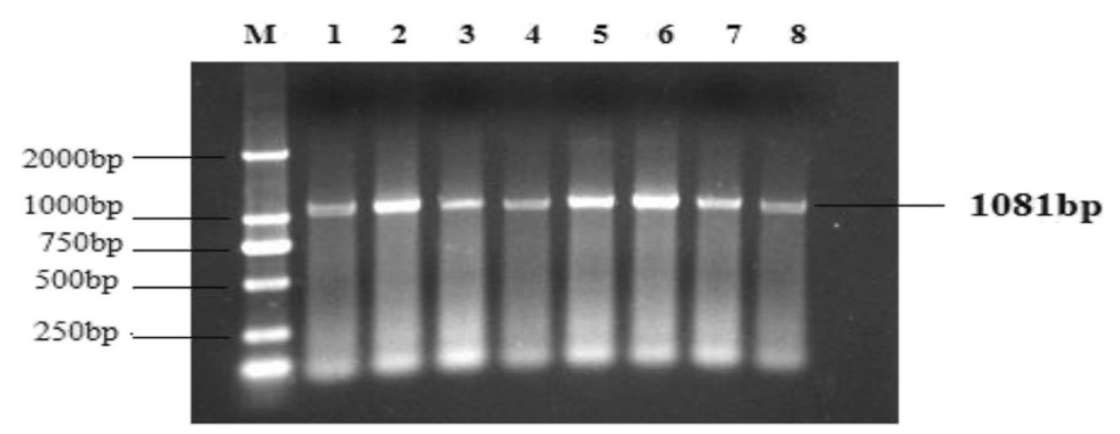

Figure 4 PCR amplification of Meq gene from tissues of chickens infected with SD $_{2012-1}$. (1) Feather pulp; (2) liver; (3) kidney; (4) muscular stomach; (5) mesentery; (6) duodenum; (7) heart; (8) bursa of Fabrius.

of CU-2 and CVI988 which had a piece of ammino acid insertion resulting suppression of Meq expression [21,22]. The Meq amino acid of SD2012-1contains a basic leucine zipper (bZIP) domain at the $\mathrm{N}$ terminal closely resembling the jun/fos oncogene family [23], SD2012-1 had the highest Meq amino acid homology with LS at $99.4 \%$, and had the lowest amino acid homology with CVI988 at 96.5\%. Like the 11 compared reference strains from China in Table 2, SD2012-1 had the same amino acid mutation of Meq gene at position 71 (S to $\mathrm{A}$ ), 77 (K to $\mathrm{E}$ ), 80 (D to $\mathrm{Y}$ ), 115 (V to $\mathrm{A}$ ), 119 ( $\mathrm{R}$ to $\mathrm{C}$ ), 153 (Q to $\mathrm{P}$ ), 176 (P to $\mathrm{R}$ ) and 217 (P to A ), which displayed regularity of strains isolated from China [11,12], except no mutation of SD2012-1 at position 139 (remain $\mathrm{T}$ no $\mathrm{A}$ ), and threonine $(\mathrm{T})$ was also found in GA, Md5, RB1B, 648A, 584A strain at position 139. It was reported that amino acid change at position 77 ( $\mathrm{E}$ to $\mathrm{K}$ ) was the feature of high virulent MDV strains, and a glutamic acid (E) at position 77 was associated with lower virulence [24], however, SD2012-1 and the 11 compared reference strains from China had glutamic acid (E) at position 77, and all the $12 \mathrm{MDV}$ strains from China were high virulence strains, this could demonstrate that a glutamic acid (E) at position 77 was not necessarily a feature of MDV strains of lower virulence. Like mild virulence, virulence and very virulence reference strains in Table 2, SD2012-1 had the amino acid of cysteine $(C)$ at position 119, whereas an arginine(R) to $C$ mutation at position 119 is found in US $\mathrm{vv}+$ MDVs 584A and the 648A strain [11].

vIL-8 gene was reported to have possibility to be associated with viral oncogenicity and pathogenicity [25], Comparing SD2012-1 with 8 reference strains, CVI988, CU-2, GA, RB1B, 648A, 584A, LS, LMS, showed SD2012-1 had the highest vIL-8 nucleotide sequence homology with LS, RB1B and CU-2 at 99.6\%, and had the lowest nucleotide sequence homology with CVI988 and 584A at $99.4 \%$. The amino acid of vIL-8 of SD2012-1 was 134aa long, the amino acid homology of SD2012-1 isolate with 8 strains was between 97.8-99.3\%, SD2012-1 had the highest amino acid homology with LS at 99.3\%, and had the lowest amino acid homology with CVI988 at $97.8 \%$. The vIL-8 gene amino acid sequence of CU-2, GA, RB1B, 648A, 584A were conservative without amino acid mutation between the 5 strains. SD2012-1 had the amino acid mutation of vIL-8 gene at position 4 (L to $\mathrm{S}$ ) and position 31 (D to G) compared with CU-2, GA, RB1B, 648A, 584A. It should be noticed that the amino acid mutations of vIL- 8 of SD2012-1 at position 4 and position 31 were found in most isolates from China [11], which displayed regularity of strains isolated from China [11].
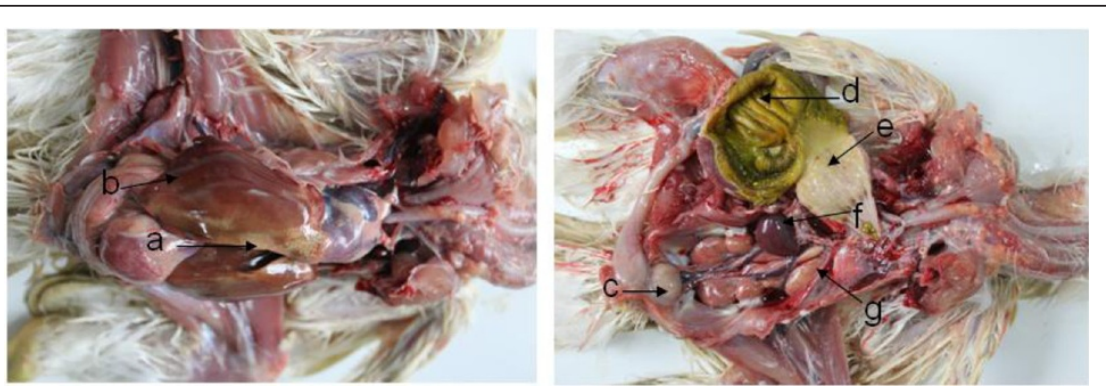

Figure 5 Pathological changes of chickens died at 55-day-old. (a) yellow degeneration diffusely in liver edge; (b) rough and uneven in liver surface; (c) bursa of Fabricius swelling; (d) muscular stomach swelling; (e) glandular stomach swelling; (f) lots of white diffused nodule in the swelled spleen; (g) kidney swelling and pale in color. 

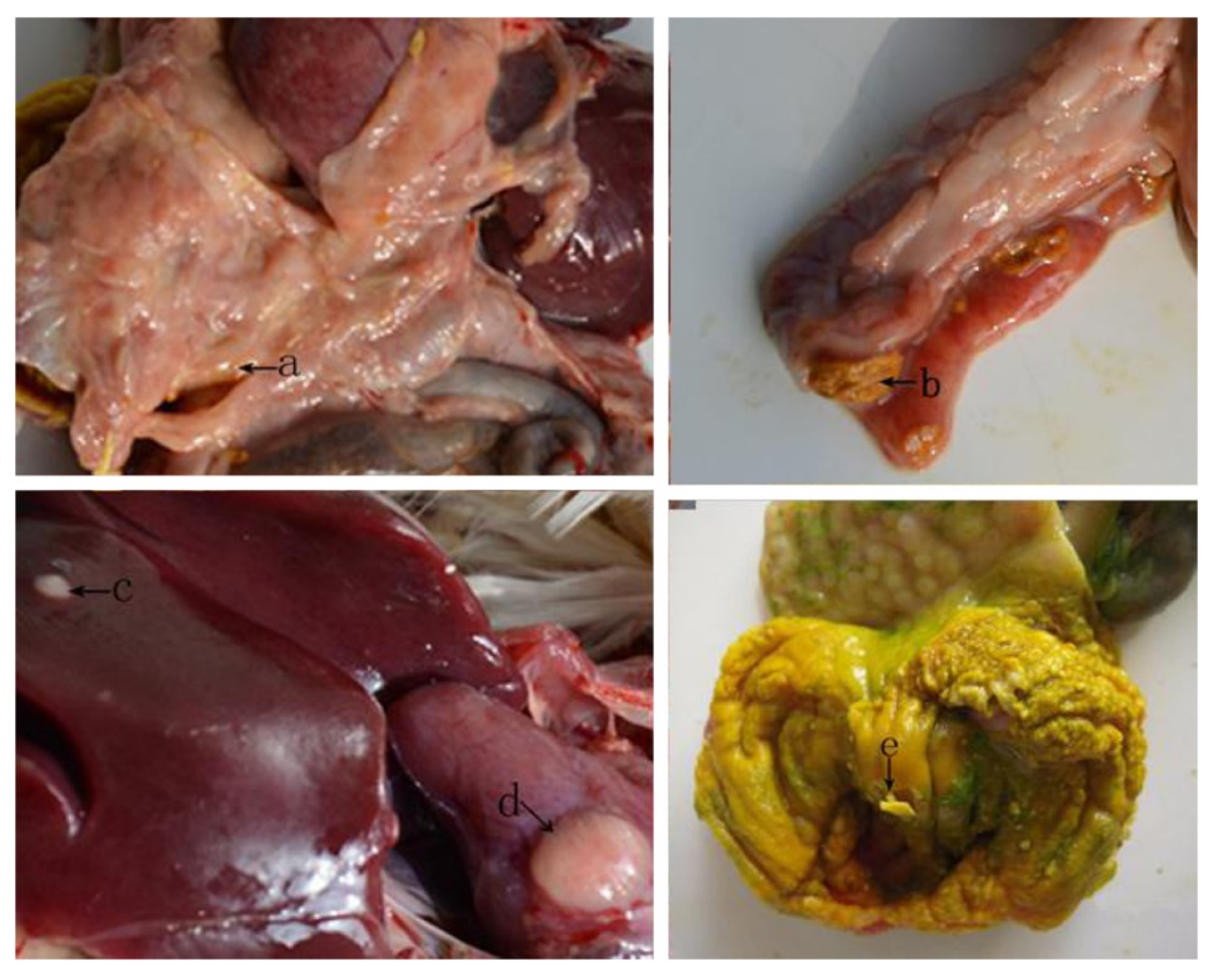

Figure 6 Pathological changes of chickens died at 90-day-old. a) diffused nodule in the thicken mesentery; (b) ulcer in duodenal mucosa; (c) tumor in liver; (d) tumor in heart; (e) muscular stomach swelling with thicken corneum layer, and easy to be peeled.

Although vaccines like HVT, HVT + SB-1 and CVI988 were widely used against Marek's disease in China, HVT was more often only used at farms for its easier storage and usage than the others, this would result in the high risks of infection with high virulent Marek's disease strains. The virulence study of SD2012-1 revealed that SD2012-1 characterized very virulence to SPF chickens, the isolate could break through the protection provided by $\mathrm{HVT}$ vaccine and $\mathrm{HVT}+\mathrm{SB}-1$ vaccine immunization and caused chicken mortality over $60 \%$, it also revealed that the immune failure occured at the farm was due to the selection of HVT vaccination. We were taking another experimental protection trial including vaccination with CVI988 against SD2012-1, the study would be reported in another paper.

Unlike LS strain isolated in Sichuan, China, which could produced high early mortality and tumor in chicken 18 days post challenge [11], SD2012-1 seemed to have a long latent period, it produced death later on 45 days post challenge in no vaccine group, and 48 days post challenge in HVT immunized group. The peak of death came on $60-85$ days post challenge.

Gross postmortem lesions were found in chickens died early with degeneration in liver, swelling of bursal and gizzard, tumors were not found in early died chickens. Although these lesions are atypical for MDV, the MDV positive detection result (Figure 4) by PCR of the organs with atypical lesion for MDV confirmed their infection with MDV. Typical MD lesion with gross tumor development was found 75 days post challenge, 30 days after the early death of MD was found, tumors could be found on liver, heart and mesentery of the later died chickens, the chickens were detected to be MDV positive by PCR.

\section{Conclusion}

In conclusion, a very virulent MDV isolate strain named SD2012-1 was isolated from a chicken flock in Shandong Province, China, which had been vaccinated with HVT vaccine, the isolate was isolated by transfecting monolayer of duck embryo fibroblast (DEF).

SD2012-1 was found to have the characteristics of very virulent MDV-1, SD2012-1 had the highest Meq amino acid homology with LS at 99.4\%, and had the lowest homology with CVI988 at 96.5\%. SD2012-1 had the same amino acid mutation of Meq gene at position 71, 77, 80, $115,119,153,176$ and 217 , which displayed regularity of strains isolated from China [11,12], except no mutation of SD2012-1 at position 139aa.

SD2012-1 had the highest vIL-8 nucleotide sequence homology with LS, RB1B and CU-2 at 99.6\%, and had the lowest homology with CVI988 and 584A at 99.4\%. SD2012-1 had the amino acid mutation of vIL-8 gene at position 4 (L to $S$ ) and position 31 (D to $G$ ), which displayed regularity of strains isolated from China [11]. 
SD2012-1 could break through the protection provided by HVT vaccine and HVT + SB-1 vaccine immunization and caused chicken mortality over $60 \%$, and the immune failure occured at the farm could be due to the selection of HVT vaccination.

SD2012-1 seemed to have a long latent period, it produced death later on 45 days post challenge in no vaccine group, and 48 days post challenge in HVT immunized group. The gross postmortem lesions of chickens challenged by SD2012-1 died early and later were different, the main gross lesion of the early died chickens were degeneration and swelling of organs without development of tumor, and gross tumor lesion occurred in organs and tissue was found later.

A better immunization way should be chosen to prevent infection of this MDV strain in field.

\section{Competing interests}

The authors declare that they have no competing interests.

\section{Author's contributions}

ZG, LZ: carried out studies design, experiment and interpretation of the data, wrote the manuscript. JW, LC: participated in experiment and interpretation of the data. HS, ZW, HM: participated in studies design and draft of the manuscript. All authors read and approved the final manuscript.

\section{Acknowledgments}

This work was financially supported by Qingdao Science and Technology Program of Basic Research "prevention and diagnosis studies on infections avian tumor disease" (Grant No.11-2-4-5-(11)-jch).

\section{Author details}

'China Animal Healthy and Epidemic Center, Qingdao 266032, China.

${ }^{2}$ Qingdao Agriculture University, Qingdao 266109, China.

Received: 5 March 2013 Accepted: 15 May 2013

Published: 20 May 2013

\section{Reference}

1. Witter RL: Increased virulence of Marek's disease virus field isolates. Avian Dis 1997, 41:149-163.

2. Osterrieder N, Kamil JP, Schumacher D, Tischer BK: Trapp S. Marek's disease virus from miasma to model. Nat Rev Microbiol 2006, 4:753-761.

3. Bulow W, Biggs PM: Differentiation between strains of Marek's disease virus and turkey herpesvirus by immunofluorescence assays. Avian Pathol 1975, 4:133-146.

4. Witter RL: Characteristics of Marek's disease viruses isolated from vaccinated commercial chicken flocks: association of viral pathotype with lymphoma frequency. Avian Dis 1983, 27:113-132.

5. Witter RL, Calnek BW, Buscaglia C, Gimeno IM, Schat KA: Classification of Marek's disease viruses according to pathotype: philosophy and methodology. Avian Pathol 2005, 34:75-90.

6. Rispens BH, van Vloten H, Mastenbroek N, Maas HJ, Schat KA: Control of Marek's disease in the Netherlands. I. Isolation of an avirulent Marek's disease virus (strain CVI 988) and its use in laboratory vaccination trials. Avian Dis 1972, 16:108-125.

7. de Boer GF, Groenendal JE, Boerrigter HM, Kok GL, Pol JM: Protective efficacy of Marek's disease virus (MDV) CVI-988 CEF65 clone C against challenge infection with three very virulent MDV strains. Avian Dis 1986 30:276-283.

8. Burgess SC, Young JR, Baaten BJ, Hunt L, Ross LN, Parcells MS, Kumar PM, Tregaskes CA, Lee LF, Davison TF: Marek's disease is a natural model for lymphomas overexpressing Hodgkin 's disease antigen (CD30). Proc Natl Acad Sci U S A 2004, 101:13879-13884.

9. Schumacher D, Tischer BK, Teifke JP, Wink K, Osterrieder N: Generation of a permanent cell line that supports efficient growth of Marek's disease virus (MDV) by constitutive expression of MDV glycoprotein E. J Gen Virol 2002, 83:1987-1992.

10. Chen M, Payne WS, Hunt H, Zhang H, Holmen SL, Dodgson JB: Inhibition of Marek's disease virus replication by retroviral vector-based RNA interference. Virology 2008, 377:265-272.

11. Tian M, Zhao Y, Lin Y, Zou N, Liu C, Liu P, Cao S, Wen X, Huang Y: Comparative analysis of oncogenic genes revealed unique evolutionary features of field Marek's disease virus prevalent in recent years in China. Virol J 2011, 8:121-131.

12. Zhang Y, Liu C, Zhang F, Shi W, Li J: Sequence analysis of the Meq gene in the predominant Marek's disease virus strains isolated in China during 2006-2008. Virus Genes 2011, 43:353-357.

13. Smith EJ, Williams SM, Fadly AM: Detection of avian leukosis virus subgroup J using the polymerase chain reaction. Avian Dis 1998, 42(2):375-380.

14. Awad AM, Abd El-Hamid HS, Abou Rawash AA, Ibrahim HH: Detection of reticuloendotheliosis virus as a contaminant of fowl pox vaccines. Poult Sci 2010, 89:2389-2395.

15. Kross I, Davis PJ, Shilleto RW: Isolation of highly cytolytic MDV strains from Germany and Spain. Avian Pathol 1998, 27:313-315.

16. Sung HW: High virus titer in feather pulp of chickens infected with subgroup J avian leukosis virus. Avian Dis 2002, 46:517-524.

17. Dudnikova E, Norkina S, Vlasov A, Slobodchuk A, Lee LF, Witter RL: Evaluation of Marek's disease field isolates by the "best fit" pathotyping assay. Avian Pathol 2007, 36:135-143.

18. Buscaglia C, Nervi P, Risso M: Characterization of four very virulent Argentinian strains of Marek's disease virus and the influence of one of those isolates on synergism between Marek's disease vaccine viruses. Avian Pathol 2004, 33:190-195.

19. Tan J, Cooke J, Clarke N, Tannock GA: Optimization of methods for the isolation of Marek's disease viruses in primary chicken cell cultures. J Virol Methods 2008, 147:312-318.

20. Teng L, Wei P, Song Z, He J, Cui Z: Molecular epidemiological investigation of Marek's disease virus from Guangxi, China. Arch Virol 2011, 156:203-206.

21. Chang K, Ohashi K, Onuma M: Diversity (polymorphism) of the Meq gene in the attenuated Marek 's disease virus (MDV) serotype 1 and MDVtransformed cell lines. J Vet Med Sci 2002, 64:1097-1101.

22. Lee LF, Wu P, Sui D, Ren D, Kamil J, Kung HJ, Witter RL: The complete unique long sequence and the overall genomic organization of the GA strain of Marek's disease virus. Proc Natl Acad Sci U S A 2000, 97:6091-6096.

23. Jones D, Lee L, Liu J, Kung HJ, Tillotson JK: Marek's disease virus encodes a basic-leucine zipper gene resembling the fos/jun oncogenes that is highly expressed inlymphoblastoid tumors. Proc Natl Acad Sci U S A 1992, 89:4042-4046

24. Shamblin CE, Greene N, Arumugaswami V, Dienglewicz RL, Parcells MS: Comparative analysis of Marek's disease virus (MDV) glycoprotein-, lytic antigen pp 38- and transformation antigen Meq-encoding genes: association of Meq mutations with MDVs of high virulence. Vet Microbiol 2004, 102:147-167.

25. Izumiya $\mathrm{Y}$, Jang $\mathrm{H}$, Ono M, Mikami T: A complete genomic DNA sequence of Marek's disease virus type 2, strain HPRS24. Curr Top Microbiol Immunol 2001, 255:191-221.

doi:10.1186/1743-422X-10-155

Cite this article as: Gong et al: Isolation and analysis of a very virulent Marek's disease virus strain in China. Virology Journal 2013 10:155. 